\title{
Analysis of Factors Affecting Road Traffic Accidents in North Cyprus
}

\author{
Mehmet Angin \\ Department of Civil Engineering \\ Near East University \\ Nicosia, Cyprus \\ mehmet.angin@neu.edu.tr
}

\author{
Shaban Ismael Albrka Ali \\ Department of Civil Engineering \\ Near East University \\ Nicosia, Cyprus \\ shabanismael.albrka@neu.edu.tr
}

\begin{abstract}
Road traffic accidents are a global issue. North Cyprus exhibits a similar trend to the rest of the world. Urbanization and the increasing number of vehicles come along with the increase of road traffic accidents. The objectives of the current study are to draw attention to the issue of road traffic accidents and to identify which factors are the main causes of road traffic accidents in North Cyprus. This article follows a two-stage study to examine the causes of this critical issue. In the first stage, a survey was conducted to determine local public opinions. The participants were asked about how environmental factors, vehicle factors, human factors, and road factors impact accidents. According to the survey outcomes, human factors and road factors have the most significant impact. In the second stage of the study, official data from official statistics were considered. The number of accidents, fatalities, and injured people are indicated initially followed by the causes of accidents. Negligent driving and speeding were identified to be the most frequent causes within the studied period. At the end of the study, the common causes of accidents in official statistics and in survey results were specified. Negligent driving and over-speeding were the major causes of road traffic accidents according to the results.
\end{abstract}

Keywords-accidents; environmental factors; vehicle factors; human factors; road factors

\section{INTRODUCTION AND BACKGROUND}

Road Traffic Accidents (RTAs) represent an important and well-known global problem. They are considered a substantial public health threat in terms of injuries, disabilities, and fatalities. Deaths caused by RTAs have increased to 1.35 million a year, while up to 50 million injuries are caused by such incidents. In other words, approximately 3,700 people lose their lives every day in RTAs. RTAs are the eighth leading cause of death for people of all ages and the leading cause of death for children and young adults between 5 and 29 years of age. The death rates are three times higher in low-income countries than in high-income countries according to a global status report on road safety published in 2018 [1]. Globally, the total number of vehicles has increased during the last decade [2]. The increase in vehicle numbers and growth in urbanization have led to traffic congestion in urban centres and subsequently to the occurrence of more RTAs. The importance of traffic safety measures has emerged as a result of the increasing number of vehicles as well as the growth in Corresponding author: Mehmet Angin urbanization [3]. Moreover, the problem of improving road quality and all concerned traffic safety equipment is a key factor of minimizing RTAs [4]. On the other hand, it has been proven that stress and environmental factors play a vital role in causing RTAs. Other important factors such as vehicle age, human error, safety measures and place and time of accident decide the seriousness of the accidents [5]. Another previous study presents an alternative solution that smartphone-based road accident determination and reporting systems can improve drivers' awareness on road safety and save lives in road accidents [6].

In North Cyprus, 478 people lost their lives and 12,551 were injured as a result of RTAs between 2007 and 2018 [712]. This study aimed to discover the main causes of RTAs in North Cyprus. The research aim was addressed by taking into consideration a combination of local public opinions and real data. The current article includes three main sections. The first section introduces the status of RTAs globally and provides different examples of past studies about RTAs. In the second section, the study area and the acquisition of data are defined. The concluding aspects are presented in the last section, in which all human and road factors were identified to have a significant impact on RTAs in the survey. On the other hand, the individual, vehicle, and environmental factors were found to have variable impact. Negligent driving and over-speeding came to the fore according to the official statistics. Most of the RTAs were recorded in 2008 and most of the accidents that caused fatalities and injuries happened in 2012 based on the same statistics.

Authors in [13] conducted a study in Turkey in which they used multinomial logit analysis to determine the risk factors that influence the severity of traffic injuries. The results showed that factors such as primary-educated drivers, singlevehicle accidents, drivers over the age of 65 , accidents occurring on provincial roads, highways, or state routes and the existence of pedestrian crosswalks increase the likelihood of fatal injuries. Moreover, accidents with private vehicles or cars reach a peak level in the evenings. Fatal injuries decrease in the presence of traffic lights or under clear weather conditions on local city streets. Authors in [14] found that general driving errors and casual pedestrian behavior cause deaths or injuries as a result of RTAs. However, they emphasized in the 
importance of road safety education. Authors in [15] analyzed both severe and non-severe RTAs on dry and wet highways. They used crash records of the 2010-2014 period. They used four different sets of crash data obtained from urban highways and four-lane rural roads in the state of Alabama. The four datasets include severe crashes on wet pavements, non-severe crashes on wet pavements, severe crashes on dry pavements and non-severe crashes on dry pavements. It was found that the rate at which younger drivers were involved in crashes was higher than the one of older drivers, except for non-severe crashes that occurred on wet pavements. In terms of the correlation between non-severe and severe crashes and gender, crash involvement rates for younger and older female drivers were found to be higher on wet pavement surfaces than dry pavement surface conditions.

Authors in [16] examined the relationships between drunk driving or being under the influence of drugs and driver-related risk factors, which are known as factors that contribute to fatal road traffic crashes. Norwegian road traffic crash registries and forensic toxicology databases were utilized. The relation between impairment and driver-related risk factors according to specific substance groups was calculated. According to the study, drug/alcohol use among car or van drivers is associated with speeding, not having a valid driver license, and not using a seatbelt. Moreover, a relationship was found between motorcycle/moped drivers and not having a valid driver license as well as not using a helmet.

Authors in [17] analyzed RTAs involving young drivers in urban areas. The study aimed to demonstrate measures that would reduce the number of RTAs involving young drivers. The authors stated the importance of starting to instil knowledge of traffic culture from an early age in order to develop safe, defensive, and attentive drivers. The educational process results in the creation of responsible drivers, which will also reduce the frequency of RTAs and indirectly the number of fatalities and casualties.

Authors in [18] studied the impact of various risk factors on RTAs. The authors aimed to identify the impact of different risk factors on RTAs in the city of Shenyang, China and discuss the different common factors that affect non-motor vehicle and pedestrian accidents. Time of year, administrative division, and age were the three most common factors for pedestrian and non-motor vehicle accidents. Factors affecting pedestrian and non-motor vehicle accidents also had different orders of importance. The authors used data from 1,227 traffic crashes in which pedestrians and drivers of non-motor vehicles were victims in Shenyang between 2015 and 2017. Administrative division, age, and season were the most common influencing factors. For pedestrian accidents, the season was the most important influencing factor, whereas for non-motor vehicle accidents, the administrative division was the most important influencing factor. Authors in [19] published an article about public health issues connected to road traffic crashes. The study analyzed the factors that contribute to road traffic crashes and made some recommendations.

\section{Methodology}

\section{A. Study Area and Data Collection}

Cyprus is an island situated in the Eastern Basin of the Mediterranean Sea [20]. North Cyprus amounts to around one third of the island, and is divided into six districts: Nicosia, Famagusta, Kyrenia, Morphou, Trikomo, and Lefka [21]. Official statistics books were used to determine the actual number of accidents, number of injured people and number of fatalities. Additionally, a survey was conducted via Google Forms to obtain the thoughts of the local community regarding RTAs. The survey included personal information, trip characteristics, and suggested accident reasons. The evaluation of the survey results and then the compiled official data are presented in this research paper. At the end of the study, the compatibility between public opinion and the actual data is presented.

\section{B. Questionnaire Design}

The survey was conducted using an online method and questions were asked to members of the local community including pedestrians, passengers, and drivers. A total of 114 people participated in the survey. Three different groups of questions were included in the survey, which included personal information, daily trip characteristics and causes of RTAs above. The first two sections of the survey include short answers or multiple-choice type questions. The third part of the survey asked the respondents to state the degree of impact (1=no impact, $2=$ impact, $3=$ great impact) of the RTA causes. The last part of the survey is divided into four sections, namely human factors, vehicle factors, road factors, and environmental factors. Each section has its own specific questions. Participants were asked to choose the most appropriate impact factor based on their aspects.

\section{Personal Information}

The personal information section is the first step of the survey. It includes questions about age, gender, job, education level, and workplace location. The respondents were in different age categories. The youngest respondents were 19 years old, and the oldest was 69 years old. The majority of the respondents were 22 years old $(9.50 \%)$. On the other hand, in terms of the gender of the participants, $68.80 \%$ of the respondents were males and $31.20 \%$ of them were females. The respondents were from different occupational fields and educational levels. The last question of the personal information part is about the workplace location. This question is a multiple-choice question with the possible choices of Nicosia, Famagusta, Kyrenia, Morphou, Trikomo, or Lefka, and not towns or villages. Although the majority of the participants worked in Nicosia, people who worked in other cities also participated in the survey, except for Trikomo, see Figure 1. Therefore, the opinions of people working in different districts were obtained through this study.

\section{Trip Characteristics}

This section includes workdays per week, time taken to drive to the workplace, and driving experience. Each participant wrote his/her own answers. Afterwards, all the provided responses were reviewed and clarified with the 
following distributions. The majority of the respondents stated that they worked 5 days per week with a ratio of $45.20 \%$. This may be a sign that many of the participants constantly encounter traffic. The second question of the trip characteristics was about trip time. It was a short-answer type of question. Thus, each person wrote a different duration. According to the responses, the maximum duration was seven and a half hours and the minimum was a minute. In the last part of the trip characteristics section, the driving experience of the respondents was examined. The driving experience distribution of the respondents is shown in Figure 2.

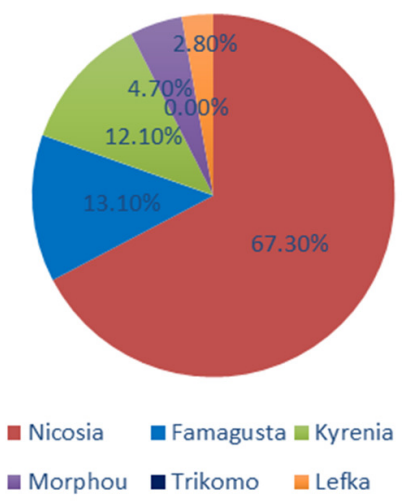

Fig. 1. Workplace distribution of the respondents.

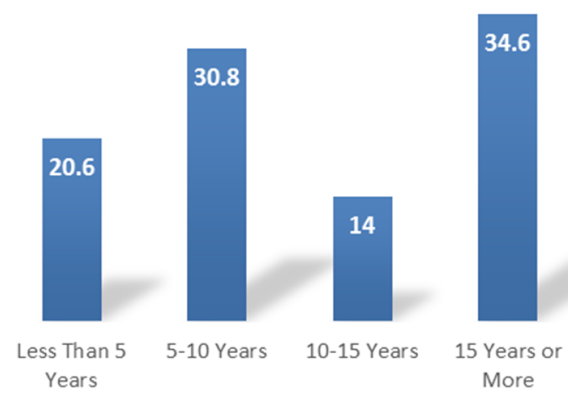

Fig. 2. Driving experience distribution of the respondents.

\section{E. Road Traffic Accident Causes}

This part of the survey is directly related to the detection of the causes of RTAs. The survey participants chose the impact factor that suited their views. Each cause has three different types of options, namely no impact, impact, and great impact, as mentioned in the questionnaire section. The first part of this subsection is about human factors, which includes negligent driving, drunk driving, inexperienced drivers, over-speeding, driving while sleepless and tired, and using a mobile phone while driving. All these reasons were considered to be risk factors that may cause RTAs and were regarded by the respondents as having a significant impact on RTAs. It should be noted that this category achieved the highest vote ratio. The distribution of these reasons is shown in Figure 3. Negligent driving has the highest percentage with $80.20 \%$. Therefore, this demonstrates the awareness among the local community about the risks of negligent driving effect in terms of crashes. Despite negligent driving having the highest percentage, over-speeding, and using a mobile phone while driving have an effect on RTAs. Over-speeding was selected by $78 \%$ and using a mobile phone while driving was chosen by $76.6 \%$ of the respondents with regard to having a significant impact. Furthermore, drunk driving and driving while sleepless or tired worsened the human-induced risk factors on RTAs. The effect of inexperience on road traffic accidents received the least percentage.

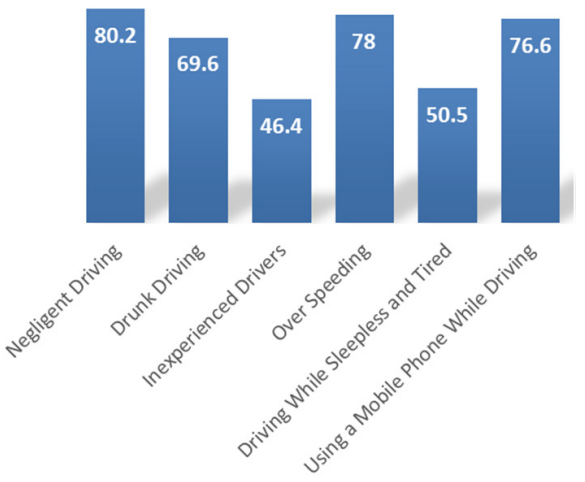

Fig. 3. Human factors distribution of the respondents.

The second part of the causes of the accidents in the survey considers vehicle factors. Those factors include bad tire usage, insufficient lighting, breakdown of engines, breakdown of gearboxes, and poor brakes. Three types of impact factors were observed for different reasons in this section. Figure 4 describes the moderate impact distribution of bad tyre usage and insufficient lighting. The bad tire usage had the highest percentage with $50 \%$. On the other hand, insufficient lighting had a moderate impact on RTAs with $44.1 \%$.

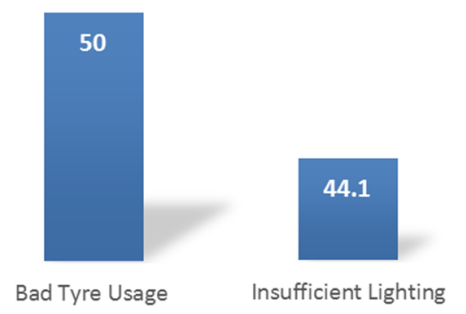

Fig. 4. Moderate impact vehicle factors.

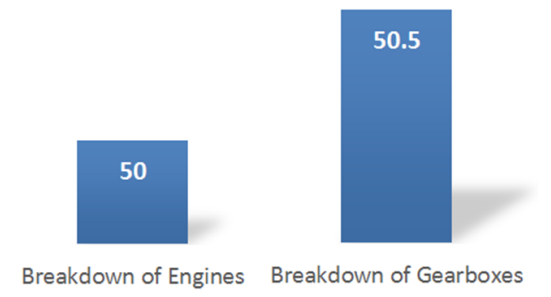

Fig. 5. No impact vehicle factors.

Furthermore, the breakdown of engines and gearboxes were considered to be factors that have no impact on RTAs according to the results. The results displayed in Figure 5 show 
the distribution of these factors. The breakdown of gearboxes has the highest percentage with $50.50 \%$ while the breakdown of engines received $50 \%$ with regard to having no impact. Lastly, poor brakes was the only factor that had a great impact for vehicles in RTAs according to the survey with a percentage $42.70 \%$.

The third part of the survey was based on road factors. The great impact choice was selected for all the reasons. Poor road surface, poor road lighting, poor road signing, speed humps and roadway geometry, are included in this part. With $68.50 \%$, poor road lighting had the highest impact percentage among these reasons. The distribution of the answers is shown in Figure 6. The last part of the survey is concerned with environmental factors. The participants were asked about the impact degree of slick road surfaces and weather conditions. According to the results, slick road surfaces have a great impact with $49.10 \%$ and weather conditions have a moderate impact with $50 \%$.

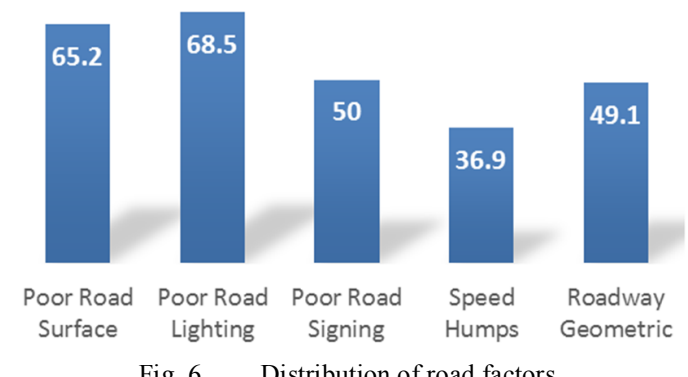

Fig. 6. Distribution of road factors.

\section{F. Road Traffic Accident Data}

In this section of the study, the actual data of the North Cyprus collected via official statistics are presented. The data period is from 2007 to 2018 . The official accident reasons are presented to be compared with the survey outcomes. Regarding the data, the number of RTAs, number of fatalities and injuries were obtained. Most of the traffic accidents occurred in 2008, whereas most of the fatal accidents and most injuries happened in 2012, as shown in Figures 7 and 8. There are fluctuations across the time period.

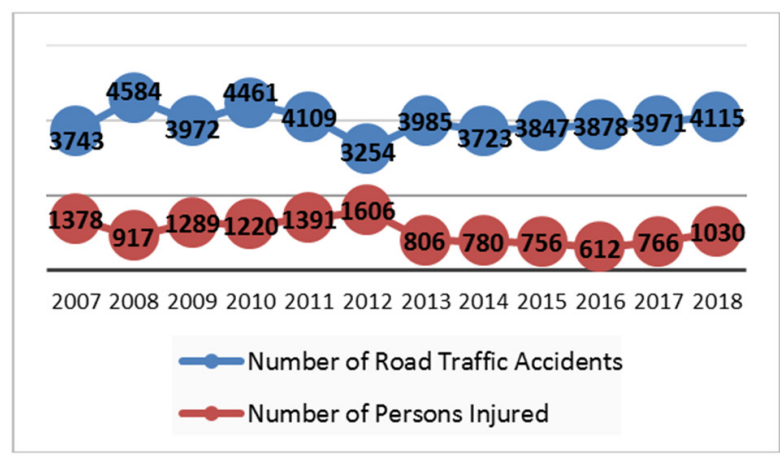

Fig. 7. Number of RTAs and injuries between 2007-2018.

Figures 9-11 exhibit all the factors that contributed to RTAs in North Cyprus with consideration of the actual data of the
2007 - 2018 period. Negligent driving, speeding, and drunk driving are dominant factor, as were and in the survey part of the study. Figures 8-10 also show the striking effect of lack of compliance with traffic signs and not keeping a safe distance while driving on RTAs.

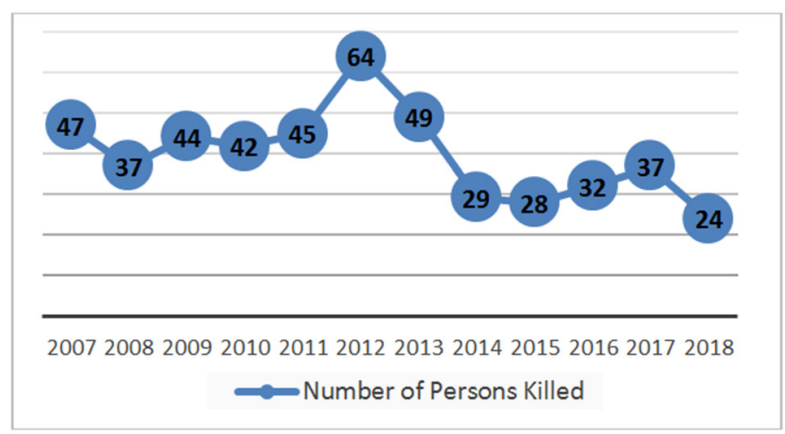

Fig. 8. Number of fatalities between 2007-2018.

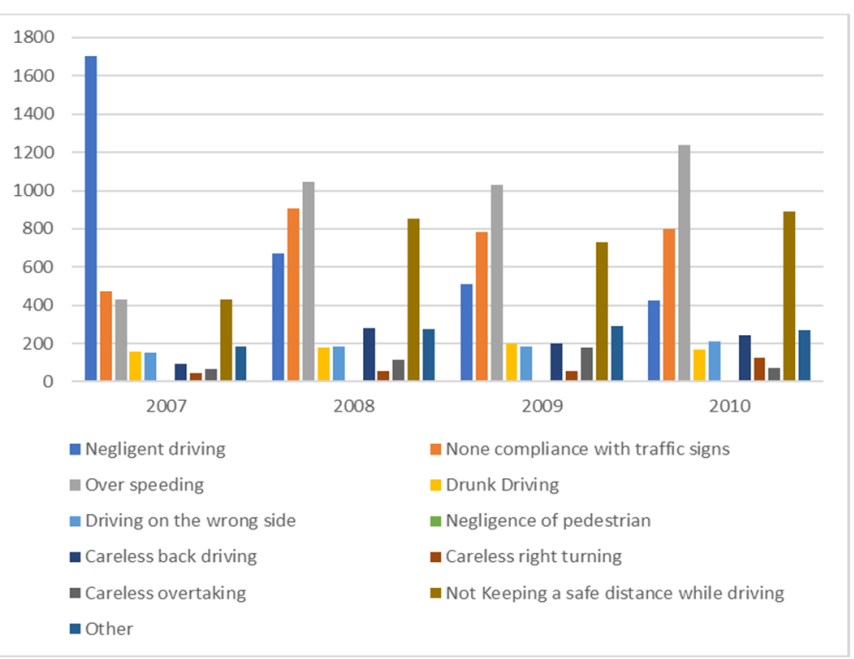

Fig. 9. Accident reasons between 2007-2010.

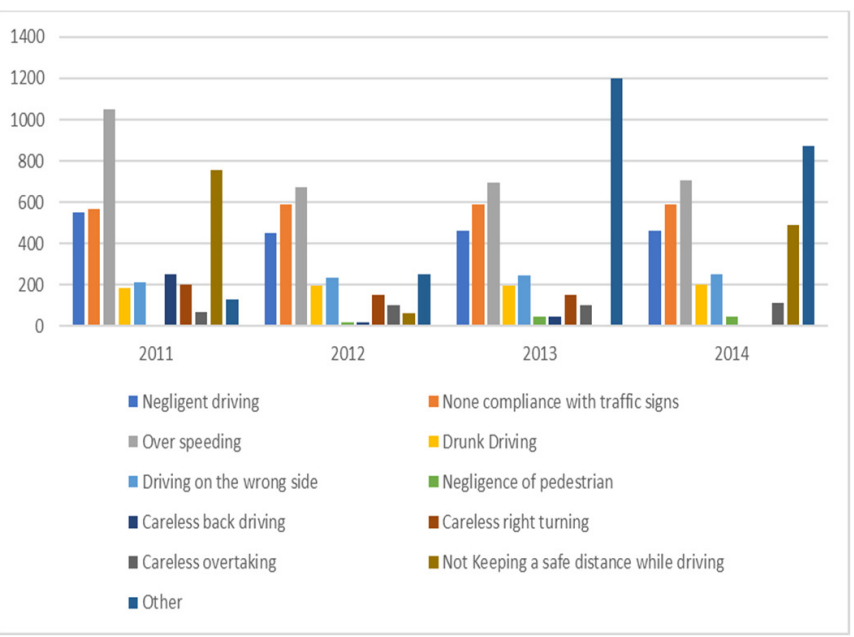

Fig. 10. Accident reasons between 2011-2014. 


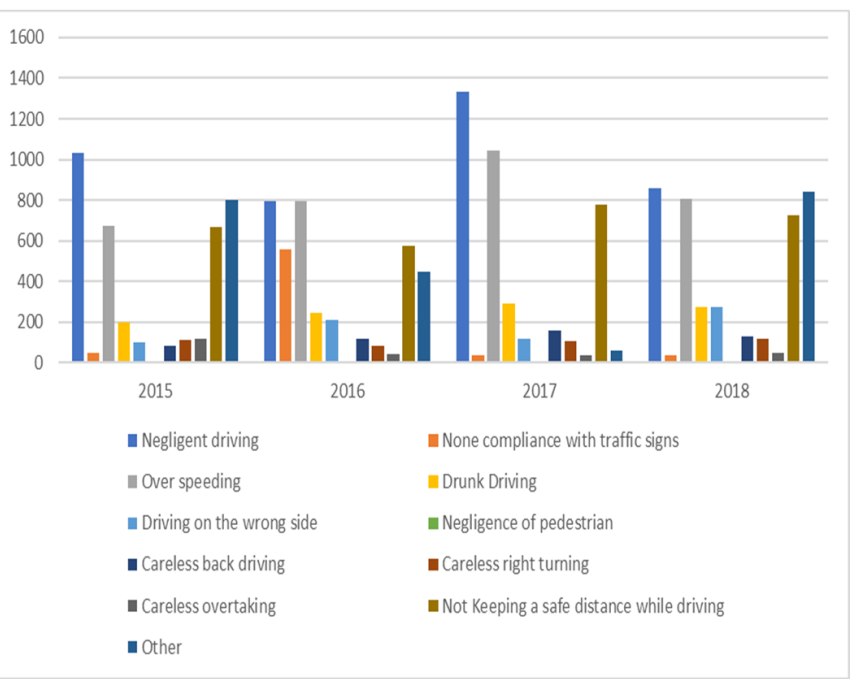

Fig. 11. Accident reasons between 2015-2018.

\section{DISCUSSION AND CONCLUSIONS}

The current study explores different factors to determine the reasons for RTAs in North Cyprus. The necessary information was obtained by conducting an online survey and researching official statistics. As a result of the evaluation of these two sources, the main risks that cause RTAs have been identified. The fact that the statistical data and the results of the survey are in accordance shows the awareness of the local community about RTAs. In addition, this situation increases the reliability of the study. The opinions of 114 respondents who answered the survey's questions were presented with charts. Human and road factors are the primary reasons for RTAs based on the conducted online survey. Human factors such as negligent driving, over-speeding, and using a mobile phone while driving were selected as primary. Other factors like poor road surface and poor road lighting are seen as the most important road factors. Furthermore, poor brakes under vehicle factors and slick roads under environmental factors were evaluated as the main causes of RTAs in North Cyprus.

According to the recorded data, RTAs increased in 2018 compared to 2007, and the number of accidents causing injuries and fatalities decreased. When we examine the distribution of accident causes in the graphs, we see that the major factors that cause accidents are negligent driving, noncompliance with traffic signs, over speeding and not keeping a safe distance while driving.

When both aspects are examined, it is observed that negligent driving and over-speeding, are common. These are the main factors that cause RTAs in North Cyprus. The results of this study can be used by policy makers to ensure traffic safety.

Based on the study findings, the following conclusions can be made:

- Human factors are the major causes of RTAs.

- Consistency of the survey and real data has been observed. Negligent driving and over speeding were major reasons in both parts of the research. Thus, the main causes of traffic accidents in North Cyprus are negligent driving and over speeding.

- Vehicle factors have 3 types of impact factors on RTAs based on the conducted survey result.

- Road factors have a great impact on the RTAs from the perspective of the North Cyprus society.

- Environmental factors have great or moderate impact on RTAs from the perspective of the local public.

- The number of RTAs increases continuously since 2014 .

- There was a decrease in RTA-related fatalities and injuries in 2018 compared to 2007 in North Cyprus.

- This study could be used for developing new mitigation strategies about RTAs in North Cyprus. These strategies can achieve success via education, awareness, and engineering solutions.

\section{REFERENCES}

[1] Global status report on road safety 2018. Geneva, Switzerland: World Health Organization, 2018.

[2] M. U. Farooq, A. Ahmed, S. M. Khan, and M. B. Nawaz, "Estimation of Traffic Occupancy using Image Segmentation," Engineering, Technology \& Applied Science Research, vol. 11, no. 4, pp. 7291-7295, Aug. 2021, https://doi.org/10.48084/etasr.4218.

[3] A. C. Igboanugo and E. F. Ekhuemelo, "Intervention Analysis of Road Traffic Accidents in Nigeria," Advanced Materials Research, vol. 18-19, pp. 375-382, 2007, https://doi.org/10.4028/www.scientific.net/AMR.1819.375 .

[4] M. Touahmia, "Identification of Risk Factors Influencing Road Traffic Accidents," Engineering, Technology \& Applied Science Research, vol. 8, no. 1, pp. 2417-2421, Feb. 2018, https://doi.org/10.48084/etasr.1615.

[5] P. Muthusamy, M. Rajendran, R. Kasimani, and P. Sivaprakash, "'A Review on Road Traffic Accident and Related Factors,'" International Journal of Applied Engineering Research, vol. 10, no. 11, pp. $28177-$ 28183, Jan. 2015.

[6] I. J. Mrema and M. A. Dida, "A Survey of Road Accident Reporting and Driver's Behavior Awareness Systems: The Case of Tanzania," Engineering, Technology \& Applied Science Research, vol. 10, no. 4, pp. 6009-6015, Aug. 2020, https://doi.org/10.48084/etasr.3449.

[7] Statistical Yearbook 2018. Nicosia, Cyprus: North Cyprus Statistical Institute, 2019

[8] Statistical Yearbook 2011. Nicosia, Cyprus: North Cyprus Statistics and Research Department, 2015.

[9] Statistical Yearbook 2016. Nicosia, Cyprus: North Cyprus Statistics and Research Department, 2017.

[10] Statistical Yearbook 2015. Nicosia, Cyprus: North Cyprus Statistics and Research Department, 2017.

[11] Statistical Yearbook 2017. Nicosia, Cyprus: North Cyprus Statistics and Research Department, 2018.

[12] Statistical Yearbook 2014. Nicosia, Cyprus: North Cyprus Statistics and Research Department, 2016.

[13] A. K. Çelik and E. Oktay, "A multinomial logit analysis of risk factors influencing road traffic injury severities in the Erzurum and Kars Provinces of Turkey," Accident Analysis \& Prevention, vol. 72, pp. 6677, Nov. 2014, https://doi.org/10.1016/j.aap.2014.06.010.

[14] J. Singh, M. K. Sahni, S. Bilquees, S. M. S. Khan, and I. Haq, "Reasons for road traffic accidents victims' perspective," International Journal of Medical Science and Public Health, vol. 5, no. 4, Jan. 2016, Art. no. 1, https://doi.org/10.5455/ijmsph.2016.07112015357. 
[15] A. Kassu and M. Anderson, "Analysis of severe and non-severe traffic crashes on wet and dry highways," Transportation Research Interdisciplinary Perspectives, vol. 2, Sep. 2019, Art. no. 100043, https://doi.org/10.1016/j.trip.2019.100043.

[16] A. Valen et al., "Driver-related risk factors of fatal road traffic crashes associated with alcohol or drug impairment," Accident Analysis \& Prevention, vol. 131, pp. 191-199, Oct. 2019, https://doi.org/10.1016/ j.aap.2019.06.014.

[17] P. Brlek, L. Krpan, I. Cvitković, and K. Lukačić, "Analysis of traffic accidents of young drivers in urban areas and measures to increase safety," Put I Saobracaj, vol. 66, no. 1, pp. 25-28, Mar. 2020, https://doi.org/10.31075/PIS.66.01.05.

[18] J. Wang, H. Lu, Z. Sun, T. Wang, and K. Wang, "Investigating the Impact of Various Risk Factors on Victims of Traffic Accidents," Sustainability, vol. 12, no. 9, Jan. 2020, Art. no. 3934, https://doi.org/ $10.3390 /$ sul2093934.

[19] J. Kulharni, "Public Health Issue Related to Road Traffic Crashes (RTCs)," International Journal of Collaborative Research on Internal Medicine \& Public Health, vol. 13, no. 2, pp. 1-6, 2020.

[20] "Geography of Cyprus," Wikipedia. Nov. 18, 2021, Accessed: Nov. 23, 2021. [Online]. Available: https://en.wikipedia.org/w/index.php?title =Geography_of_Cyprus\&oldid=1055817966.

[21] "Cyprus," Wikipedia. Nov. 05, 2021, Accessed: Nov. 23, 2021. [Online]. Available: https://en.wikipedia.org/w/index.php?title=Cyprus\&oldid= 1053625350 . 